Review Article

\title{
Impact of NGS in the medical sciences: Genetic syndromes with an increased risk of developing cancer as an example of the use of new technologies
}

\author{
Pablo Lapunzina ${ }^{1,2}$, Rocío Ortiz López ${ }^{3,4}$, Lara Rodríguez-Laguna ${ }^{2}$, Purificación García-Miguel ${ }^{5}$, \\ Augusto Rojas Martínez ${ }^{3,4}$ and Víctor Martínez-Glez ${ }^{1,2}$ \\ ${ }^{1}$ Centro de Investigación Biomédica en Red de Enfermedades Raras, Instituto de Salud Carlos III, \\ Madrid, Spain. \\ ${ }^{2}$ Instituto de Genética Médica y Molecular, Hospital Universitario la Paz, Madrid, Spain. \\ ${ }^{3}$ Departamento de Bioquímica y Medicina Molecular, Facultad de Medicina, \\ Universidad Autónoma de Nuevo León. Monterrey, Nuevo León, México. \\ ${ }^{4}$ Centro de Investigación y Desarrollo en Ciencias de la Salud, Universidad Autónoma de Nuevo León, \\ Monterrey, Nuevo León, México. \\ ${ }^{5}$ Unidad de Oncología Pediátrica, Hospital Infantil La Paz, Madrid, Spain.
}

\begin{abstract}
The increased speed and decreasing cost of sequencing, along with an understanding of the clinical relevance of emerging information for patient management, has led to an explosion of potential applications in healthcare. Currently, SNP arrays and Next-Generation Sequencing (NGS) technologies are relatively new techniques used to scan genomes for gains and losses, losses of heterozygosity (LOH), SNPs, and indel variants as well as to perform complete sequencing of a panel of candidate genes, the entire exome (whole exome sequencing) or even the whole genome. As a result, these new high-throughput technologies have facilitated progress in the understanding and diagnosis of genetic syndromes and cancers, two disorders traditionally considered to be separate diseases but that can share causal genetic alterations in a group of developmental disorders associated with congenital malformations and cancer risk. The purpose of this work is to review these syndromes as an example of a group of disorders that has been included in a panel of genes for NGS analysis. We also highlight the relationship between development and cancer and underline the connections between these syndromes.
\end{abstract}

Keywords: NGS, new technologies, developmental syndrome, cancer predisposition.

\section{Introduction}

Genetic testing using new technologies, such as Next-Generation Sequencing (NGS), is transitioning from the research to the diagnostic phase. Methodologies for translating new technologies to the clinical setting are not well established and are currently in the difficult process of transitioning from a primarily research environment and culture to a more quality controlled diagnostic environment. This significant change is having an enormous impact on health, but it is also creating diagnostic dilemmas. The rapid development of NGS has radically reduced both the cost and time required for exome or genome analysis and has allowed for the implementation of panels of genes for diagnosis and research in both genetic syndromes and cancer.

Send correspondence to Víctor Martínez-Glez. Instituto de Genética Médica y Molecular, Hospital Universitario la Paz, Instituto de Salud Carlos III, Paseo de la Castellana 261, 28046 Madrid, Spain. E-mail: vmartinezglez@salud.madrid.org.
The impact of genetics and genomics in health

During the last two decades, developments in genetics and enormous advances in genomic technologies have altered the capability of understanding diseases, making diagnoses and providing effective clinical management. From a mechanistic point of view, genetic disorders may be roughly subdivided in three main subtypes: genomic, genetic and epigenetic. Genomic disorders encompass those diseases in which there is either a gain or loss (sometimes both) of genomic material. They usually include very large gains and losses, such as those that are observed in trisomies and monosomies, as well as smaller, cryptic, medium-size aberrations, such as those that are observed in microduplication and microdeletion syndromes. Although there is no absolute consensus, the term genomic disorders applies to rearrangements of between $5 \mathrm{~kb}$ and several megabases. Genetic disorders refer to small gains and losses, usually of 1-5000 bp. Epigenetic disorders are those without gains and losses that have chemical changes (often 
methylation defects but also acetylation as well as others). Considering these disorders together ( $\sim 8000)$, genomic disorders account for $\sim 8-12 \%$ of the cases, genetic disorders $\sim 85-87 \%$ and epigenetic disorders $\sim 2-3 \%$. The current vision of genomics in healthcare and public health represents a confluence of the development of three important strands: genetic technologies, clinical genetics and genomic healthcare. Genetic technologies encompass the whole range of laboratory technologies that provide detailed sequence and other genomic information, whether related to an individual's germ-line or somatic cells, that is, the altered genome within cancer cells.

The explosion of potential applications in healthcare has arisen from the increased speed and decreasing cost of sequencing, along with an increased understanding of the clinical relevance of emerging information for patient management. Currently, SNP arrays and NGS technologies are relatively new techniques used to scan the genome for gains and losses and losses of heterozygosity (LOH), SNPs and indel variants. SNP arrays are proving to be useful tools in the detection of chromosomal imbalances and $\mathrm{LOH}$ in a wide range of disorders (Miller et al., 2010), and NGS provides a rapid and complete sequencing of a set of candidate genes, the entire exome (whole exome sequencing, WES) and even the whole genome (WGS) (Tucker et al., 2009; $\mathrm{Ng}$ et al., 2010).

Clinical genetics is a specialty that provides services for individuals and families affected by or at risk of a genetic disorder or congenital abnormality. It includes diagnostic assessment, counseling and support, genetic testing and provision of advice to patients and their extended family. Traditionally, clinical genetics has encompassed chromosomal disorders, dysmorphic syndromes, teratogenic disorders and single gene disorders, which may be evident in childhood or later in life. The challenge that arises for clinical genetics is that many inherited disorders, including a large number of single gene disorders, manifest with a wide range of clinical symptoms; as a result, clinicians in some specialties need to be skilled in recognizing, diagnosing and managing these genetic conditions. Genomic healthcare widens the range of applications of genomic technologies to include instances where they may be used to recognize a precise molecular sub-type of disease and hence fine-tune treatment or determine disease susceptibility.

\section{The impact of new technologies in genomic medicine}

The prevailing rhetoric amongst basic grant funding bodies and sponsors, researchers and many policy-makers worldwide is that genomic medicine represents a revolution in healthcare, with some even referring to it as "genomic tsunami." It is envisaged that the use of genomic technologies to enable patient diagnosis and treatment based on information about a person's entire DNA se- quence, through WGS, or the portion of the genome that encodes proteins, through WES, will become a part of mainstream healthcare practices. Genomics is having an impact in many areas of clinical medicine, but it is not so much a revolution as it is an evolution. Clinical research leaders are slowly gaining knowledge and experience with these approaches, and the process of embedding new practices in high quality care pathways throughout our country is gradual and difficult.

New technologies and clinical knowledge have facilitated significant progress in the capability of clinicians to diagnose and manage genetic and heritable disorders arising in a wide range of clinical areas. It is believed that this will be rapidly followed rapidly a burgeoning of "genomic medicine" in which a wider analysis of genomic information will be used to predict, prevent, diagnose and treat many common chronic and rare disorders. It is important that the development and configuration of clinical and laboratory genetics and other specialties is optimized to meet the expected future capacity and range of needs. Such optimization should build on the strengths of existing structures and processes and should aim to incorporate genomics into existing clinical pathways. It has been recommended that relationships between specialty genetic services and a range of other clinical specialties should be formed as a key foundation for the development of genomic medicine.

The rapid development of NGS has radically reduced both the cost and the time required for WES and WGS. The Human Genome Project took approximately 13 years to sequence the first human genome and cost several billion dollars; today, the same process can be completed within weeks for a few thousand dollars using NGS. Soon it may be easier and cheaper to sequence an entire genome than to sequence a single gene or genotype a series of known mutations. Furthermore, using the same technologies, gene expression profiling and epigenetic analyses are becoming simpler and cheaper. WES and WGS together with our evolving knowledge of genes and disease are likely to change the current practice of medicine and public health by facilitating more accurate, sophisticated and costeffective genetic testing. WES and WGS have several important clinical applications in the short to medium term: improved diagnosis and management of diseases with a strong heritable component as well as personalized diagnosis and stratified treatment of cancer through tumor profiling. Over the long term, these technologies may also have many other applications, including tissue matching, risk prediction and pharmacogenetics.

Cancer is one of the most prevalent groups of diseases and is associated with high morbidity and mortality. Sporadic Cancer is defined as neoplasias that have a monoclonal origin in somatic cells and arise in a person without family history or any known predisposing genetic alteration. When cancer occurs in family members more frequently than the general population it is known as Familial 
or Hereditary Cancer and is associated with germ-line DNA alterations that increase the risk of carcinogenesis.

Although the cause of most childhood cancers remains unknown, it has been established that the risk is higher in children with congenital anomalies and some specific genetic syndromes. These Cancer Predisposition Syndromes are generally caused by germ-line mutations in specific genes that can predispose family members to develop cancer at an early age. These syndromes are rare, and it has traditionally been estimated that tumors arising in this context represent $5-10 \%$ of childhood tumors; however, this number seems to be increasing (Schiffman et al., 2013).

Within cancer predisposition syndromes, there exists a subgroup characterized by the presence of multiple congenital malformations due to developmental defects and a higher than normal risk of developing tumors. These are referred to as Genetic developmental syndromes with an increased risk to develop cancer. This group is composed of a broad spectrum of syndromes with different causes and clinical features in which the presence of tumors is not always the most characteristic manifestation. Some of the main features of these syndromes and the associated genes are listed in Table 1, and some examples of phenotypic appearance are shown in Figure 1.

The purpose of this work is to review the main genetic syndromes associated with congenital malformations and increased cancer risk as an example of a group of disorders that has been included in a panel of genes used for NGS analysis. We also highlight the relationship between development and cancer, and underline the connections between these syndromes.

\section{Cancer-Related and Syndrome-Causing Genes}

Recently, studies of genes known to cause genetic syndromes in the context of their involvement in neoplastic processes have led to a better understanding of both cancer and rare diseases. Nevertheless, little is known regarding how defects in these genes and in signaling pathways can produce congenital developmental abnormalities as well as alterations underlying the development of neoplastic process. It seems that the relationship between development and cancer could be the result of inversely related events. During embryonic development, genetic and epigenetic instructions direct growth and differentiation with an orderly and well-established pattern, while oncogenic alterations lead to undifferentiated cells in a disorganized and chaotic pattern (Bellacosa, 2013).

The occurrence of tumors in genetic developmental syndromes depends on many variables: the molecular pathway involved (differentiation, proliferation, cell death, etc.), the affected gene (oncogene, tumor suppressor or DNA repair gene), the type of alteration (genetic, genomic, or epigenetic), and the origin of these changes (germ-line or somatic). This occurrence also explains why a large propor- tion of developmental defects associated with cancer can be grouped into a few clusters with overlapping clinical manifestations, common molecular pathways, and similar pathogenic mechanisms (Figure 2).

\section{Overgrowth syndromes, RASopathies, PTENopathies and AKT/PI3K/mTOR pathway}

As shown in Figure 2, these syndromes are represented by four different clusters but are described here in a single section because they are closely related and share many nodes.

Overgrowth Syndromes (OGS) are one of the main groups of cancer-related developmental syndromes. OGS are composed of a heterogeneous group of disorders in which the main feature is that weight, height, and/or head circumference are 2-3 standard deviations above the mean for the patient's sex and age. In some OGS, tumors appear mostly in the abdominal region, usually before 10 years of age. Beckwith-Wiedemann syndrome shows a $10 \%$ cumulative risk of cancer at 4 years of age; the associated cancers are generally Wilms tumor and hepatoblastoma, but adrenal neuroblastoma and rhabdomyosarcoma have been described. In Perlman syndrome, only cases of Wilms tumor and/or bilateral renal hamartomas have been reported, while in Sotos syndrome, lympho-hematologic malignancies are more common, followed by Wilms tumor, sacrococcygeal teratoma, and neuroblastoma (Lapunzina, 2005).

Within OGS there is a well-defined subgroup known as "RASopathies," characterized by variable degrees of intellectual deficit, dysmorphic face, cardiac alterations, skin manifestations, and predisposition to cancer in some cases. The RAS genes are widely known because of their activation in $20-30 \%$ of human cancers; these genes play an important role in the RAS/MAPK signaling cascade, translating extracellular growth stimuli into cellular responses, with impacts on cell proliferation, differentiation, and death. Therefore, it is not surprising that disruption of Ras protein function is involved in the pathogenesis of many human diseases, including cancer. In addition to somatic mutations in cancers, mutations in the RAS genes causing a dysregulation of the RAS/MAPK pathway may also occur in the germ-line or as a post-zygotic event. Such mutations lead to the appearance of RASopathies, and the phenotypic overlap among RASopathies can be explained by alteration of the same molecular pathway. RASopathies include Noonan, neurofibromatosis type 1, Costello, LEOPARD and Cardio-Facio-Cutaneous (CFC) syndromes; some of these syndromes are associated with an increased risk of developing cancer (Hafner and Groesser, 2013).

The relationship between genetic alterations and susceptibility to cancer is not always straightforward. Costello syndrome patients show tumors in $10-15 \%$ of cases diagnosed with a mutation in $H R A S$, and it has been suggested that the risk is higher in individuals with the G12A muta- 


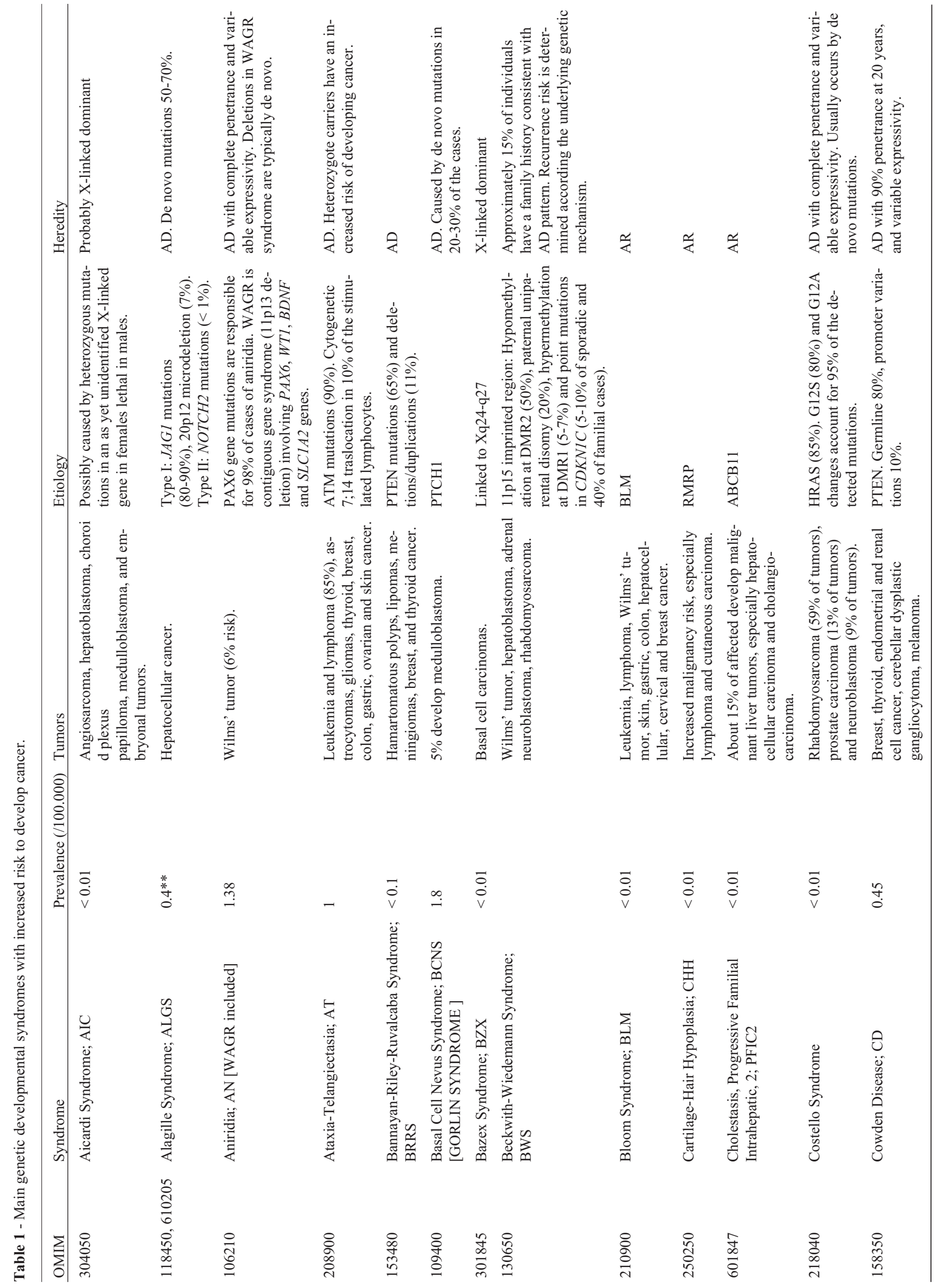



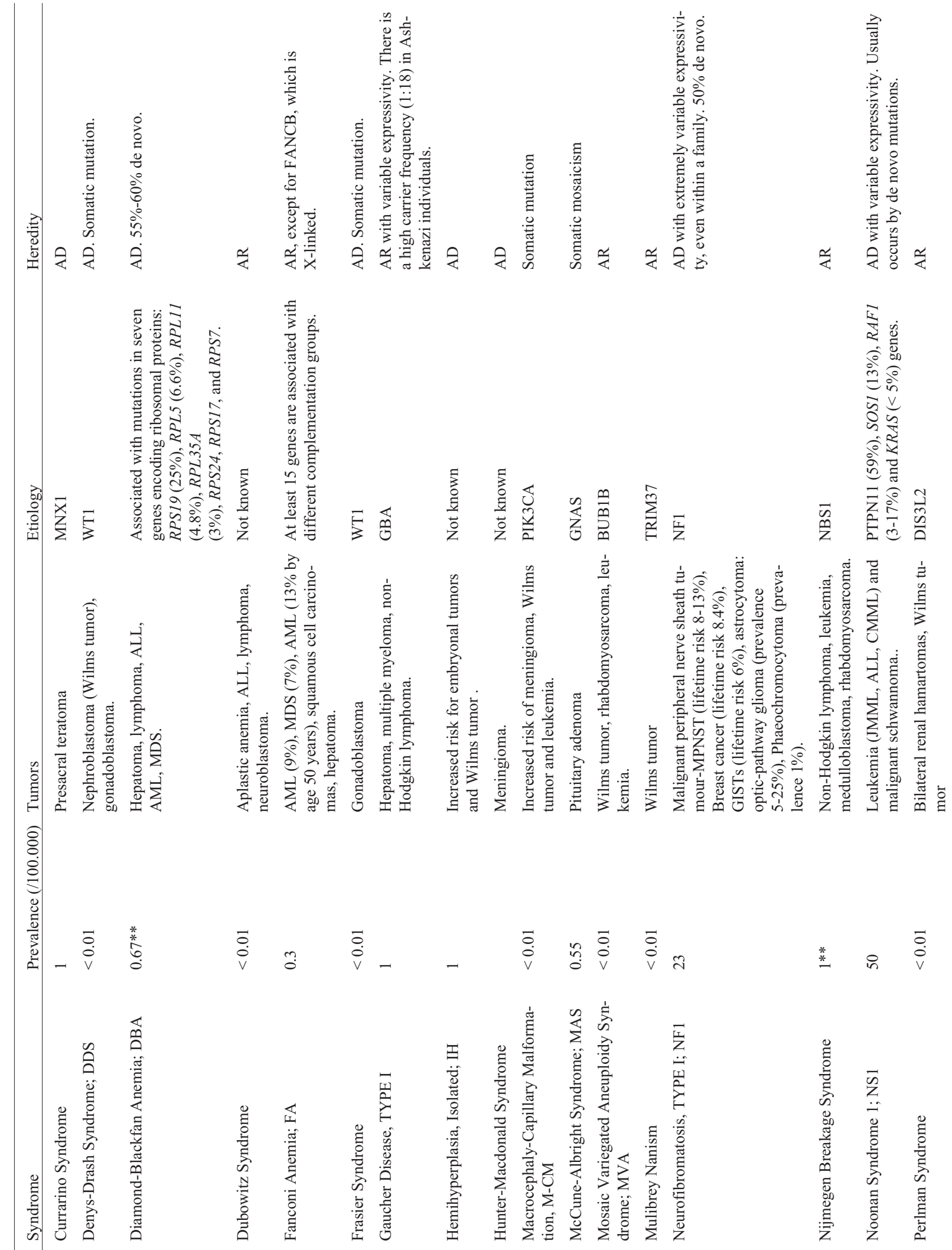

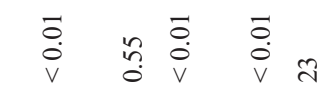

* in $\stackrel{\overrightarrow{0}}{*}$ 


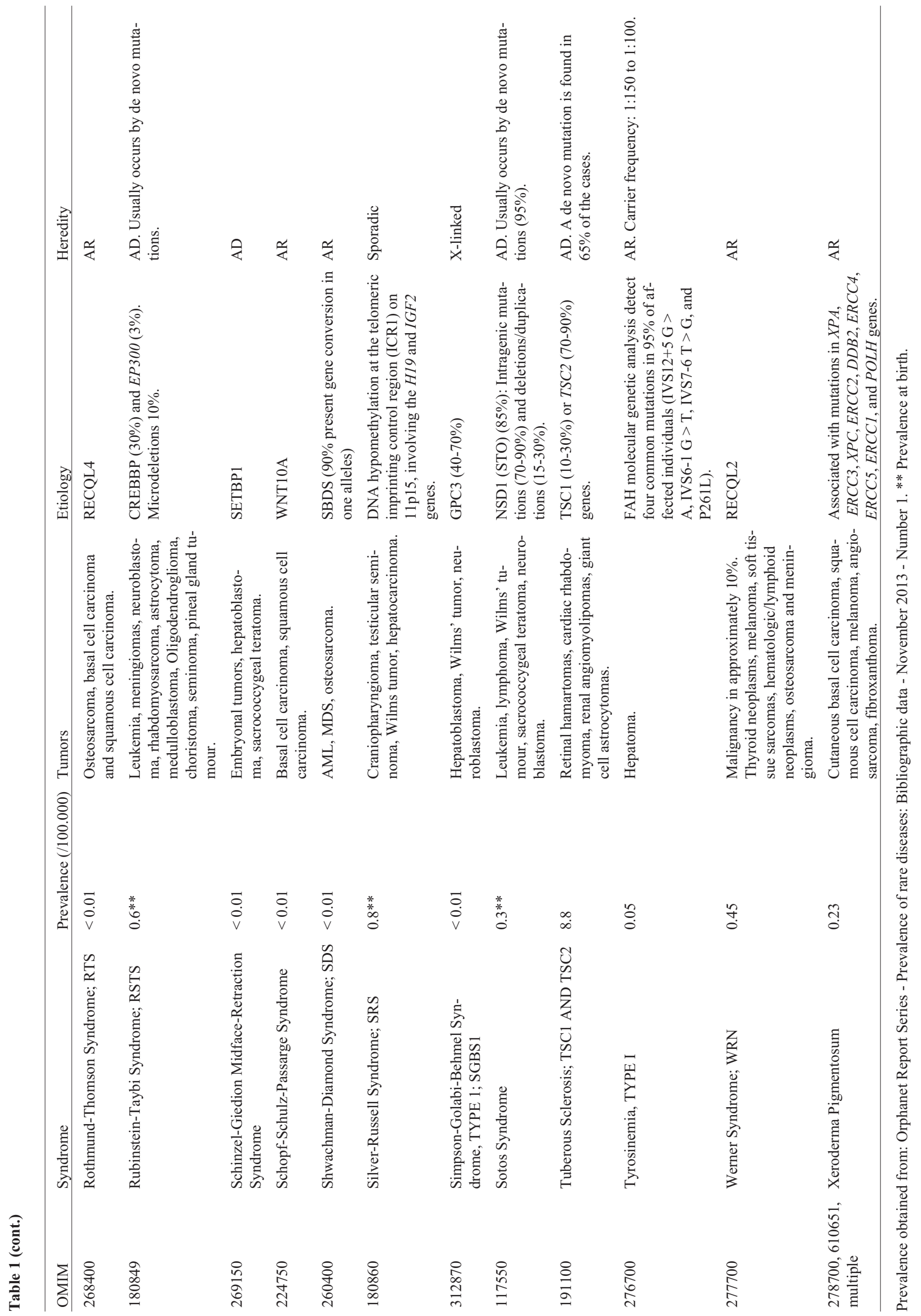




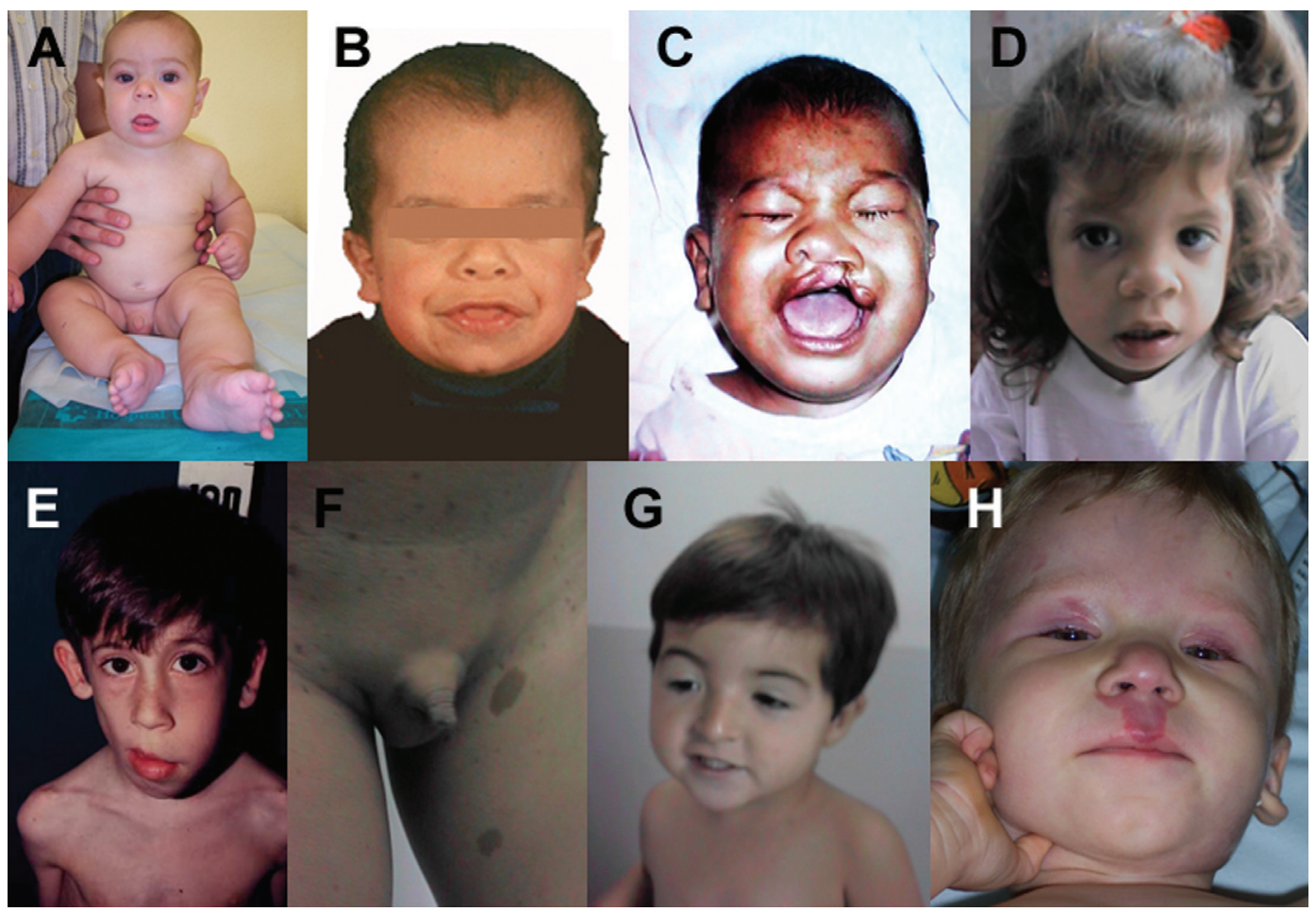

Figure 1 - Phenotypic presentations in some genetic developmental syndromes that have an increased risk of developing cancer. (A) BeckwithWiedemann. (B) Costello. (C) Simpson-Golabi-Behmel. (D) Noonan. (E) Hemihyperplasia. (F) NF1. (G) Rubinstein-Taybi. (H) MegalencephalyCapillary Malformation.

tion $(\sim 55 \%)$ than in those possessing the G12S mutation $(\sim 5 \%)$. The greatest potential for malignant transformation has been observed with the G12V mutation; it is the most common mutation in sporadic tumors, although it has not

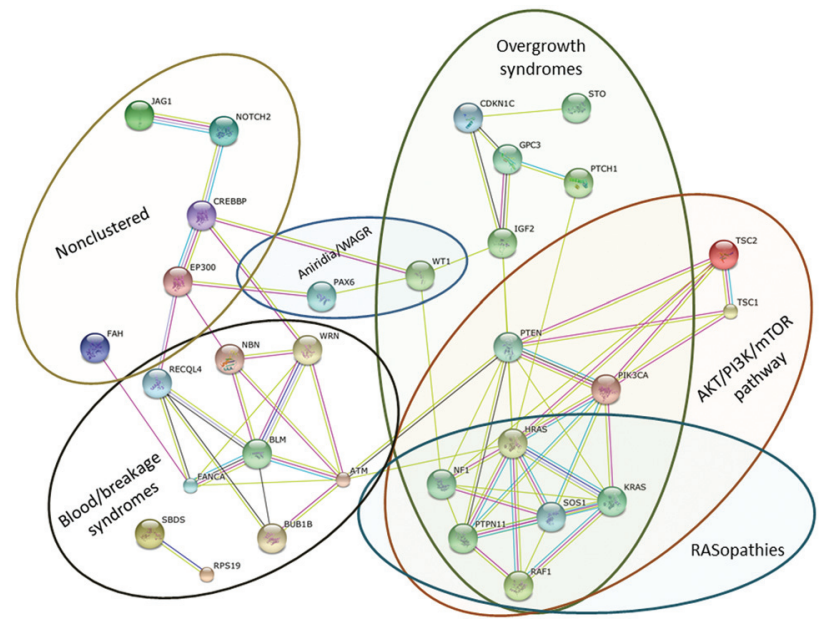

Figure 2 - Known and predicted associations between major genetic developmental syndromes with an increased risk of developing cancer using the Search Tool for the Retrieval of Interacting Genes/Proteins (STRING) database of physical and functional interactions version 9.1 (Jensen et al., 2009). Disconnected nodes are not shown. Xeroderma Pigmentosum, Diamond-Blackfan anemia and Fanconi anemia were simplified to one gene for visual purposes. Lines connecting nodes represents association due to coexpression (black), coocurrence (dark blue), experiments (pink), databases (light blue), text mining (green), and homology (purple). been detected in Costello syndrome patients (Aoki et al., 2005). In general, the spectrum of mutations causing germ-line RASopathies is different than those causing tumors; however, there is a greater overlap compared to mosaic RASophaties (Hafner and Groesser, 2013).

Another well-known tumor suppressor gene involved in the pathogenesis of developmental syndromes is PTEN (Mester and Eng, 2013). This gene modulates the AKT/PI3K/mTOR and MAPK pathways that inhibit proliferation and promote cell death. Genetic and epigenetic inactivations of PTEN are frequently found in a variety of tumor types, and PTEN mutations are responsible for the pathogenesis of the PTEN hamartoma tumor syndromes (PHTS) that are characterized by overgrowth and multiple hamartomas. PHTS include Cowden syndrome and Bannayan-Riley-Ruvalcaba syndrome, both of which demonstrate a $25-50 \%$ and $10 \%$ lifetime risk of developing breast and thyroid cancer, respectively, as well as PTEN-related Proteus syndrome and Proteus-like syndrome. PTEN is a natural inhibitor of $A K T 1$, an oncogenic kinase that acts downstream of PI3K to control proliferation and survival. Activating mutations in the $A K T 1$ gene that are likely only tolerated in a mosaic state cause Proteus syndrome, the main differential diagnosis of PHTS, and are characterized by segmental manifestations. Tumors are not the main feature of Proteus syndrome, but additional somatic mutations may lead to cancer development. 
Further downstream in the AKT/PI3K/mTOR pathway is the PIK3CA oncogene, which has been frequently implicated in various neoplasms, including breast, lung and cervical cancer. The helicase and kinase domains of this protein are hot spots for somatic mutations associated with PIK3CA gain of function mutations and cancer. Mutations in PIK3CA are also involved in segmental overgrowth syndromes, such as Megalencephaly-Capillary Malformation (M-CM), Hemimegalencephaly, and CLOVES. In M-CM patients, mutations in PIK3CA are most often de novo and somatically mosaic, suggesting that the mutation occurred in the embryo post-fertilization. Mutations in PIK3CA associated with M-CM are not usually found in cancers, contrary to what has been observed for Hemimegalencephaly and CLOVES syndromes in which mutations related to cancer in PIK3CA hot spots have been reported (Mirzaa et al., 2013).

Although it is not an OGS but still affects the AKT/PI3K/mTOR pathway, Tuberous Sclerosis Complex (TSC) is a disorder caused by inactivating mutations in either the TSC1 or TSC2 tumor suppressor genes responsible for inhibiting mTOR, another pathway often activated in human cancers. TSC is characterized by the presence of hamartomatous lesions in several organs throughout the body and is accompanied by seizures, intellectual disability, and renal and pulmonary disease. TSC tumors may develop in any tissue, including the brain (cortical tubers, subependymal nodules and subependymal giant cell astrocytomas), heart (cardiac rhabdomyoma), kidney (angiomyolipomas and renal cysts), or liver (angiomyolipomas and hepatic hamartomas) (Borkowska et al., 2011).

\section{Blood/breakage syndromes}

The stability and integrity of DNA is essential for cellular and organismal survival. Consequently, DNA repair mechanisms are mediated by multiple molecular pathways, use different enzymes to act on different injuries (environmental damage, DNA replication errors, etc.) and are essential for preventing deleterious mutations and genetic instability.

Emergence of cancers is a natural consequence of mutations to DNA repair mechanisms; however, when germ-line mutations affecting repair mechanisms occur, they give rise to complex developmental disorders characterized by premature aging and/or increased risk of cancer, such as Ataxia Telangiectasia, Bloom Syndrome, Fanconi anemia, Nijmegen Breakage syndrome, Werner syndrome, and Xeroderma Pigmentosum, the last of which leads to a 10,000 -fold increase in risk of non-melanoma skin cancer and a 2,000-fold increased risk of melanoma (Knoch et al., 2012).

$A T M$ (related to ataxia telangiectasia) is involved in damage-induced repair of specific DNA double-strand breaks (DSB) and in the activation and regulation of many pathways, including p53-dependent apoptosis. NBS1
(Nijmegen Breakage syndrome) plays an important role as a DNA DSB sensor and activator of ATM. The XP-proteins (Xeroderma Pigmentosum) are involved in nucleotide excision repair as well as in transcription as chromatin remodeling factors. WRN (Werner syndrome) and BLM (Bloom syndrome) belong to the family of RecQ helicases; the former plays multiple roles in DSB-repair, base excision repair, and telomere maintenance, while the latter is involved in damage recognition, homologous recombination, chromosome integrity, cell division, and chromatin remodeling. The genes involved in Fanconi anemia-pathway play a role in DNA repair, interstrand-crosslink repair and telomere maintenance (Knoch et al., 2012). Another developmental syndrome, Rothmund-Thomson syndrome, is also caused by mutations in a RecQ helicase (RECQL4), but it is not usually included in the breakage syndromes despite being one of its main differential diagnoses. Rothmund-Thomson syndrome is characterized by poikiloderma, telangiectasia, congenital skeletal abnormalities, premature aging, and increased risk of malignant disease, especially osteosarcoma.

Finally, we have included some disorders associated with lymphohematologic alterations in this cluster due to their characteristics and types of neoplasm. As happens in Fanconi anemia, and which are included in its differential diagnosis, Diamond-Blackfan anemia and ShwachmanDiamond syndrome are rare diseases involving bone marrow failure, congenital malformations and increased risk of developing tumors, primarily acute myelogenous leukemia, myelodysplastic syndrome and some solid tumors, such as osteogenic sarcoma. Diamond-Blackfan anemia is caused by mutations in the RPS19 gene encoding a ribosomal protein, while Shwachman-Diamond syndrome is caused by mutations in the $S B D S$ gene encoding a protein that might have roles in RNA metabolism and ribosome biogenesis.

\section{Other syndromes}

Some classic developmental syndromes do not form large evident clusters; however, they do show an increased risk of developing cancer and, in some cases, have relationships to one another, the significance of which have yet to be defined (Figure 1). The most noteworthy cluster is Aniridia/WAGR: its primary neoplasm, Wilms tumor, is present in many overgrowth syndromes, and it is directly associated with hypermethylation of the imprinting center 1 , genomic abnormalities and uniparental disomy in chromosome region 11p15, all causing Beckwith-Wiedemann syndrome.

Using the STRING database (Jensen et al., 2009) to look for physical and functional protein interactions, NOTCH2 (Alagille syndrome) and CREBBP (Rubinstein Taybi syndrome) share predicted functional links when testing for homology, searching databases and text mining. Rubinstein Taybi syndrome (CREBBP and EP300) is associated with Aniridia/WAGR (PAX6 and $W T 1$ ) by experi- 
ments and data mining and has been related to Werner $(W R N)$, Rothmund-Thomson (RECQL4) and Nijmegen Breakage (NBS1) syndromes by testing for homology, experiments, and text mining. Although the interactions predicted by this database have limitations, they are a useful starting point for future studies.

\section{Final Considerations}

Studies in patients and families with cancer predisposition and developmental syndromes have allowed us to understand not only tumors arising in hereditary cancers but also to understand cancer as a genetic disease of somatic cells. The spectrum of disorders ranging from pure developmental syndromes through intermediate states, such as cancer predisposition syndromes with a varied presence of congenital malformations to sporadic cancer, seems to be a continuous line drawn by numerous interconnected molecular pathways. A deeper understanding of these alterations is making possible the use of molecular targets associated with genetic syndromes in the treatment of sporadic tumors, and in the future, may even allow the use of the drugs used in oncology for the management of developmental disorders. (Bellacosa, 2013).

No less important, physicians and especially clinical geneticists and oncologists should be aware of the existence of these sometimes rare syndromes and their high risk of developing tumors. This knowledge is essential for making a correct diagnosis and for monitoring and treating patients. The new high-throughput technologies have enabled us to establish diagnostic and research tests for syndromes that often have overlapping clinical manifestations and are caused by multiple genes and mechanisms. With a bit of enthusiasm and determination, the expectations raised with the acquired knowledge and use of new technologies will be met in the near future.

\section{References}

Aoki Y, Niihori T, Kawame H, Kurosawa K, Ohashi H, Tanaka Y, Filocamo M, Kato K, Suzuki Y, Kure S, et al. (2005) Germline mutations in HRAS proto-oncogene cause Costello syndrome. Nat Genet 37:1038-1040.
Bellacosa A (2013) Developmental disease and cancer: Biological and clinical overlaps. Am J Med Genet A 161A:27882796.

Borkowska J, Schwartz RA, Kotulska K and Jozwiak S (2011) Tuberous sclerosis complex: Tumors and tumorigenesis. Int J Dermatol 50:13-20.

Hafner C and Groesser L (2013) Mosaic RASopathies. Cell Cycle 12:43-50.

Jensen LJ, Kuhn M, Stark M, Chaffron S, Creevey C, Muller J, Doerks T, Julien P, Roth A, Simonovic M, et al., 2009. STRING 8 - A global view on proteins and their functional interactions in 630 organisms. Nucleic Acids Res 37:D412D416.

Knoch J, Kamenisch Y, Kubisch C and Berneburg M (2012) Rare hereditary diseases with defects in DNA-repair. Eur J Dermatol 22:443-455.

Lapunzina P (2005) Risk of tumorigenesis in overgrowth syndromes: A comprehensive review. Am J Med Genet C Semin Med Genet 137C:53-71.

Mester J and Eng C (2013) When overgrowth bumps into cancer: The PTEN-opathies. Am J Med Genet C Semin Med Genet 163C:114-121.

Miller DT, Adam MP, Aradhya S, Biesecker LG, Brothman AR, Carter NP, Church DM, Crolla JA, Eichler EE, Epstein CJ, et al. (2010) Consensus statement: Chromosomal microarray is a first-tier clinical diagnostic test for individuals with developmental disabilities or congenital anomalies. Am J Hum Genet 86:749-64.

Mirzaa G, Conway R, Graham JM and Dobyns WB (2013) PIK3CA-related segmental overgrowth. In: Pagon RA, Adam MP, Bird TD, Dolan CR, Fong CT and Stephens K (eds) Gene Reviews. Univ. Washington, Seattle.

Ng SB, Buckingham KJ, Lee C, Bigham AW, Tabor HK, Dent KM, Huff CD, Shannon PT, Jabs EW, Nickerson DA, et al. (2010) Exome sequencing identifies the cause of a mendelian disorder. Nat Genet 42:30-35.

Schiffman JD, Geller JI, Mundt E, Means A, Means L and Means V (2013) Update on pediatric cancer predisposition syndromes. Pediatr Blood Cancer 60:1247-1252.

Tucker T, Marra M and Friedman JM (2009) Massively parallel sequencing: The next big thing in genetic medicine. Am J Hum Genet 85:142-54.

License information: This is an open-access article distributed under the terms of the Creative Commons Attribution License, which permits unrestricted use, distribution, and reproduction in any medium, provided the original work is properly cited. 